\title{
A Complete and the Most Liberal Semantics for Converging OR Gateways in Sound Processes
}

\author{
Thomas M. Prinz and Wolfram Amme \\ Friedrich Schiller University Jena, 07743 Jena, Germany \\ Thomas.Prinz@uni-jena.de, Wolfram.Amme@uni-jena.de
}

\begin{abstract}
Although the semantics of converging OR gateways (also known as OR-joins) in business processes is far from trivial, they are frequently used. In this paper, we describe a general definition for soundness of processes guaranteeing the absence of deadlocks and no lack of synchronization for each possible OR-join semantics. Then, we derive a criterion completeness - to evaluate existing approaches of OR-join semantics. As a result, no currently existing OR-join semantics is complete; therefore, there are actually correct processes being not successfully executable. For that, we provide our own approach based on a traditional relation of compiler construction; and we show that this approach is complete and can be called the most liberal possible.
\end{abstract}

Keywords: Business process, OR-join, completeness, semantics, soundness.

\section{Introduction}

Graphical notations of programs and processes allowing explicit parallelism, e.g., Business Process Model and Notation (BPMN) [1] or Event-driven Process Chains, are standard during the modeling of (business) processes in software engineering and business process management. Such a modeling of processes is a difficult and error-prone task. Especially, the fusion of different paths is sometimes complex, so that often it is the best choice to use an inclusive converging (OR) gateway - for reasons of correctness and readability, e.g., because of compactness. However, the semantics of the converging OR gateways, called OR-joins, is one of the major problems in defining an automatic and correct execution of arbitrary processes [2].

The major task of OR-joins in practice is the synchronization of an arbitrary non-empty set of control flows. Figure 1 illustrates two processes in BPMN notation containing OR-joins. The lefthand process $(a)$ has an OR diverging gateway $O S_{1}$ (an OR-split) making it possible to execute an individual non-empty subset of its successor tasks $T_{1}, T_{2}$, and $T_{3}$. The OR-join $O J_{1}$ then combines these flows into a single one. In this case, this OR-join only has to wait for the arrival of all incoming control flows, i.e., an OR-join has a non-local semantics since its execution does not only depend on the control of its incoming edges. Moreover, an OR-join has to wait for all control flows which may still arrive at its incoming edges. This informal description of 'may still arrive' implies possible future states. However, they can only be determined if the semantics of OR-joins exists. Therefore, a semantics of OR-joins has to be defined carefully without using future states, i.e., the state space.

A second problem is that two or more OR-joins may mutually depend on each other as one OR-join can be executed only if the other is not and vice versa. These situations are called vicious circles [3] and have high relevance in practice [2]. 

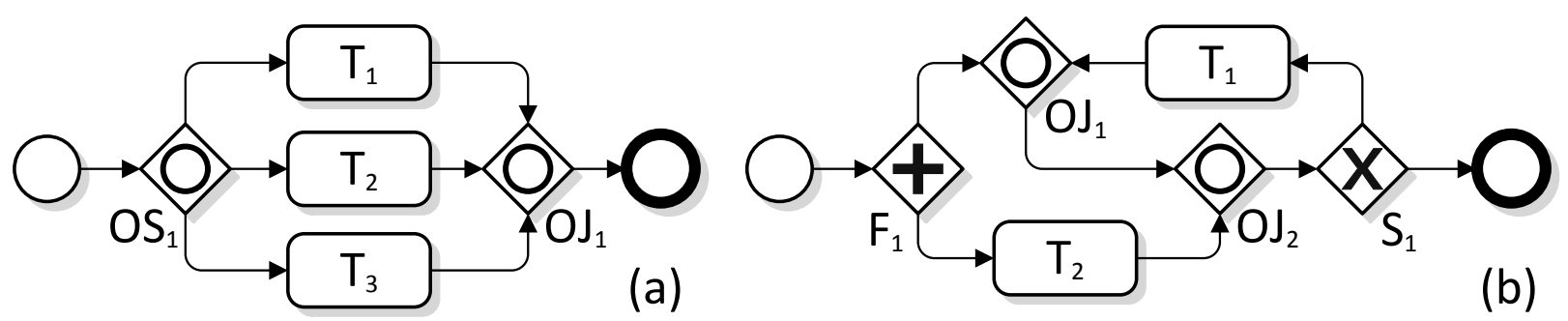

Figure 1. Two processes in BPMN notation containing OR-joins

The right-hand side $(b)$ of Figure 1 addresses the introduced difficulties (following [2]). It contains a diverging AND gateway (a fork) $F_{1}$ supplying both OR-joins $O J_{1}$ and $O J_{2}$. The simple semantics of the previous example does not hold since the OR-join $O J_{1}$ would wait for the execution of $\mathrm{OJ}_{2}$ and vice versa - a vicious circle. Such vicious circles are well-researched and there are a lot of well-established and carefully defined semantics of OR-joins, e.g., [2], [3], [4], [5], [6], [7], [8], [9], [10], with a considerable practical relevance.

However, Figure 2 illustrates a sample process which leads to failures by using state-of-the-art semantics. It describes a car service in a garage. At first, the garage receives a car for an inspection and creates an order. Afterwards, an accounting system creates the corresponding invoice and, at the same time, the inspection starts. During the inspection, the customer may have special requests, e.g., changing the oil or testing the air pressure of the tires. Those special requests would be performed at the same time like the inspection. If all requests are done, the garage cleans up the car as a special service, followed by an inspection of the customer. If the customer is unsatisfied about the cleanness of his or her car, the garage keeps cleaning the car until the customer is pleased.

If we throw away all textual information and eliminate the special request part from the sample process, we get the same abstract process as illustrated on the right-hand side of Figure $1(b)$. We will use that abstract process as counter example in Section 4 where we show that it leads to failures (and therefore cannot be properly executed) by the use of state-of-the-art semantics.

For this reason, in this paper, we provide a general definition of soundness for business processes which promises the absence of failures [11], [12], [13]. Our common definition of soundness makes it possible to define completeness, building a criterion to evaluate the quality of existing OR-join semantics with regard to the number of sound processes being successfully executable. Therefore, we will show that all existing OR-join semantics (to the best of the authors' knowledge), especially the popular approach of Völzer [2], are good approximations of a complete OR-join semantics - however none is complete, i.e., they lead to failures in sound processes as mentioned before.

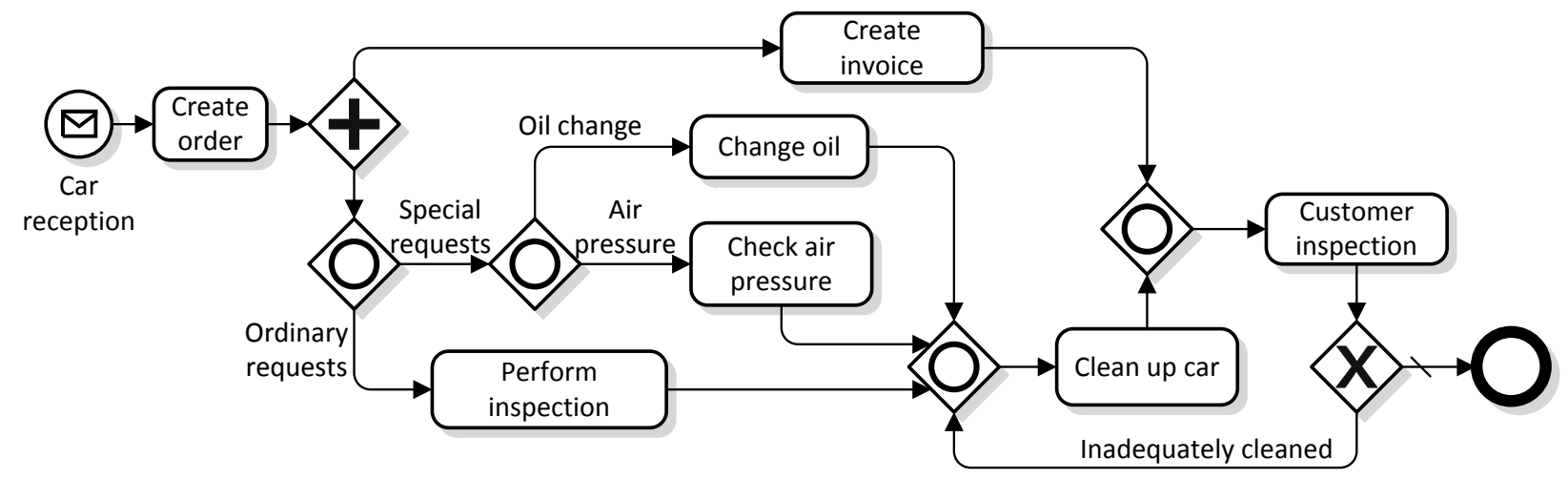

Figure 2. Car service process 


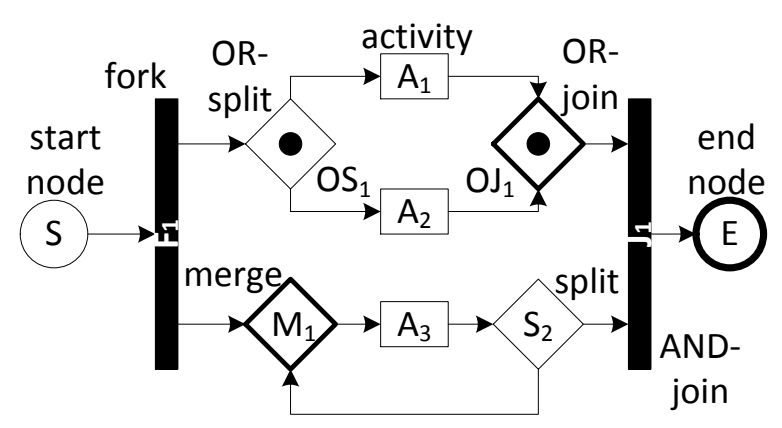

Figure 3. A workflow graph with OR-joins

The definition of a (first) complete OR-join semantics as described in this paper has several advantages: 1) The definition of an intuitive and correct semantics for process modelers is simpler if the limits of OR-join semantics are known. 2) A successful replacement of OR-joins with converging XOR gateways (merges) or converging AND gateways (AND-joins) needs completeness to be correct. 3) Although someone may argue for the poor modeling of processes with OR-joins in cases where state-of-the-art semantics do not work, it is always possible that accurately modeled business processes are not correctly executable when using OR-joins in process collaborations, in big processes, or for reasons of readability and compactness of resulting processes.

The rest of the paper is structured as follows: Section 2 summarizes some definitions and notions, followed by the definition of soundness and completeness with OR-joins in Section 3. Afterwards, we evaluate existing OR-join semantics with regard to completeness (Section 4) and introduce a first complete semantics in Section 5. The complete semantics will then be applied on two examples in Section 6. Finally, Section 7 concludes this paper.

\section{Preliminaries}

In this section, we introduce some basics of processes. Processes are mostly represented as workflow nets (Petri nets) [12] or workflow graphs being special cases of control flow graphs allowing explicit parallelism [11]. We prefer workflow graphs since the modeling of OR gateways in Petri nets is difficult [10]. Before we define workflow graphs, the term of paths is introduced. Formally, a path $P=\left(n_{1}, \ldots, n_{m-1}, n_{m}\right), m \geq 2$, for a digraph $G=(N, E)$ is a sequence of nodes of $N$ such that $\forall i \in\{1, \ldots, m-1\}:\left(n_{i}, n_{i+1}\right) \in E$. We write $n \in P$ iff $n \in\left\{n_{1}, \ldots, n_{m}\right\}$. Sometimes, we use a similar definition of paths consisting of edges and depending on the context, i.e., a path $P=\left(e_{1}, \ldots, e_{m-1}, e_{m}\right), m \geq 2$, is a sequence of edges of $E$ such that $\forall i \in\{1, \ldots, m-1\}:$ the target node of $e_{i}$ is the source node of $e_{i+1}$.

A workflow graph is a directed graph $W F G=(N, E)$ where $N$ is a set of nodes which is partitioned into disjoint sets of activities $N_{A}$, forks $N_{F}$, AND-joins $N_{J}$, splits $N_{S}$, merges $N_{M}$, OR-splits $N_{O S}, O R$-joins $N_{O J}$, and $\{$ start, end $\}$ such that (1) start is the unique start node and end is the unique end node, (2) the end node, activity, split, OR-split, and fork each have exactly one incoming edge, (3) merges, OR-joins, and AND-joins have at least two incoming edges, (4) the start node, activity, merge, OR-join, and AND-join each have exactly one outgoing edge, (5) splits, OR-splits, and forks have at least two outgoing edges, and (6) each node $n \in N$ lies on a path from the start node to the end node.

Figure 3 shows a sample workflow graph. The start and end nodes are depicted as (thick) circles, whereas an activity is depicted as a rectangle. Forks and AND-joins are illustrated as thin massive rectangles, and splits and merges as (thick) diamonds. OR-splits and OR-joins are depicted as (thick) diamonds with dots in their center.

Usually, the semantics of workflow graphs is defined, similarly to Petri nets, as a token game. Therefore, a state of a workflow graph $W F G=(N, E)$ is represented by tokens on the edges 
of the graph, i.e., a state of $W F G$ is a mapping $S: E \rightarrow \mathbb{N}$ which assigns a natural number to each edge. If $S(e)=k$, we say that edge $e$ carries $k$ tokens in state $S$. In the initial state, only the outgoing edge of the start node carries a token, whereas in the termination state, only the incoming edge of the end node carries a token. After a node $n \in N$ will be executed (fired) in state $S$, the state changes into a state $S^{\prime}$, depicted as $S \stackrel{n}{\rightarrow} S^{\prime}$. A state $S^{\prime}$ is reachable from a state $S$ (written $S \rightarrow^{*} S^{\prime}$ ) if there is a finite sequence $S \stackrel{n_{f}}{\rightarrow} S_{1} \ldots S_{k-1} \stackrel{n_{k}}{\rightarrow} S^{\prime}, k \geq 0$.

Basically, a semantics of an arbitrary node $n$ can be divided into two parts: The enabledness of $n$ in a state $S$, i.e., whether node $n$ can be executed or not, and the effect on state $S$ if $n$ will be executed. The effect is traditionally defined as a modification of the number of tokens on the incoming and outgoing edges of the executed node. An activity, a fork, and an AND-join remove one token from each of their incoming edges and add one token to each of their outgoing edges. A merge removes non-deterministically one token from one of its incoming edges carrying a token and adds one token to its outgoing edge. In workflow graphs that carry no data as, referred in this paper, a split removes one token from its incoming edge and decides non-deterministically on which of its outgoing edges it will add one token. An OR-split performs the same way as a split; however it adds one token for each edge of a non-empty subset of its outgoing edges. At this moment, it is assumed that an OR-join removes one token of all of its incoming edges carrying a token and adds one token to its outgoing edge. Vanhatalo et al. [14] formalize the effect of a node $n$ and a state change $S \stackrel{n}{\rightarrow} S^{\prime}$ (also for OR-joins) in general as follows:

1. $n$ is a fork, AND-join, or an activity:

$$
S^{\prime}(e)= \begin{cases}S(e)-1, & e \text { is an incoming } \\ & \text { edge of } n \\ S(e)+1, & e \text { is an outgoing } \\ & \text { edge of } n \\ S(e), & \text { otherwise }\end{cases}
$$

2. $n$ is a split or merge and there are an incoming $e^{\prime}$ edge with $S\left(e^{\prime}\right) \geq 1$ and an outgoing edge $e^{\prime \prime}$ of $n$ :

$$
S^{\prime}(e)= \begin{cases}S(e)-1, & e=e^{\prime} \\ S(e)+1, & e=e^{\prime \prime} \\ S(e), & \text { otherwise }\end{cases}
$$

3. $n$ is an OR-split or OR-join and there is a non-empty set Out of outgoing edges of $n$ :

$$
S^{\prime}(e)= \begin{cases}S(e)-1, & e \text { is an incoming edge of } n \text { such that } S(e) \geq 1 \\ S(e)+1, & e \in \text { Out } \\ S(e), & \text { otherwise }\end{cases}
$$

Thus, tokens (may) travel through the workflow graph depending on the nodes (possibly) firing. Moreover, we say that a token on an edge $e$ in state $S$ arrives on an incoming edge of a node $n$ (or simply arrive at $n$ ) iff the token of $e$ travels through state changes to that incoming edge of $n$.

The enabledness of a node can be seen as a mapping from a node $n$ and a state $S$ to enabled or not enabled, called release function. In general, if $\mathfrak{S}$ is the set of all possible states of $W F G$, the mapping is defined as $F: N \times \mathfrak{S} \rightarrow\{$ enabled, not enabled $\}$. To keep the release function simple, we describe only the conditions when the release function evaluates to enabled in the remainder of this paper, knowing that it evaluates to not enabled otherwise. Basically, a node is not enabled in a state $S$ if it has no incoming edge which carries a token. In the following, we call each node $n$ active in state $S$ if this basic property (that one of $n$ 's incoming edges carries a token) holds. Most nodes (activities, splits, merges, forks, and OR-splits) are actually enabled if they are active. However, an AND-join is not enabled until each of its incoming edges has a token. Since the release function of OR-joins is the focus of this paper, we refer to it simply as release function in the following and introduce some approaches in Section 4. 


\section{Soundness, Completeness, and Liberty}

Soundness, introduced by van der Aalst [15], is one of the most frequently used correctness criterion of business processes and guarantees the absence of deadlocks and lack of synchronization if data is not considered. A state $S$ of $W F G$ is a deadlock iff there is a token on an incoming edge of a node $n$ such that each reachable state from $S$ carries also a token on that edge. We also say that $n$ causes a deadlock. $S$ has a lack of synchronization iff there is an edge which carries more than one token in $S$. Usually, a workflow graph is called sound iff no deadlock or state with a lack of synchronization is reachable from the initial state.

Since the release function of OR-joins is currently undefined, we cannot compute reachable states from an initial state. Therefore, we define soundness regarding OR-joins in its most general manner:

Definition 1 (Soundness). Let $W F G=(N, E)$ be a workflow graph.

$W F G$ is sound iff there is a release function F of OR-joins such that from the initial state neither a deadlock nor a state with a lack of synchronization is reachable. In this case, we say that $F$ holds for WFG.

This definition has the following advantages:

(1) The definition is equal to classical soundness iff the workflow graph has no OR-joins since each arbitrary release function can be used.

(2) If the workflow graph is unsound without regarding the release function, it is unsound for each arbitrary release function.

(3) If and only if there is a sound (non-deterministic) execution of the workflow graph, there is at least one valid release function.

We have a look at the four workflow graphs of Figure 4 as examples for this general definition of soundness. The workflow graph $(a)$ is sound as there exists a simple release function $F$ for the OR-joins that does not result not in a deadlock nor in a lack of synchronization, e.g., waiting for all tokens on those edges which have a path to it. The workflow graph $(b)$ is naturally not sound as there is no release function that can avoid the possible lack of synchronization in the merge $M_{1}$, above all as the workflow graph has no OR-joins. Workflow graph $(c)$ is not sound either and workflow graph $(d)$ is sound. The reason why the last both workflow graphs are (not) sound is explained in the following, especially, in Section 5.

With the help of this general definition of soundness, we can define a criterion - completeness - to evaluate the quality of a release function.

Definition 2 (Completeness). Let $\mathfrak{W} \mathfrak{F} \mathfrak{G}$ be the set of all sound workflow graphs and $\mathfrak{F}$ the set of all release functions holding for each $W F G \in \mathfrak{W} \mathfrak{F} \mathfrak{G}$.

A release function $F$ is complete iff $F \in \mathfrak{F}$. We say that a complete release function is a most liberal one.

\section{Evaluation}

In the last section, we have defined the notion of completeness which allows the evaluation of existing OR-join semantics approaches with regard to their liberalness. For this, we introduce a sample workflow graph (cf. Figure 5) being used as a counterexample to show that existing release functions are not as liberal as possible. If this workflow graph will be executed, it starts its execution with a single token on the incoming edge of the fork $F_{1}$. Since that fork is then enabled, it fires and the activity $A_{2}$ can be executed so that the OR-joins $O_{1}$ and $O_{2}$ have a token on exactly one of their incoming edges $e_{1}$ and $e_{3}$ in a state $S$. Now, there are four possibilities in $S$ : 


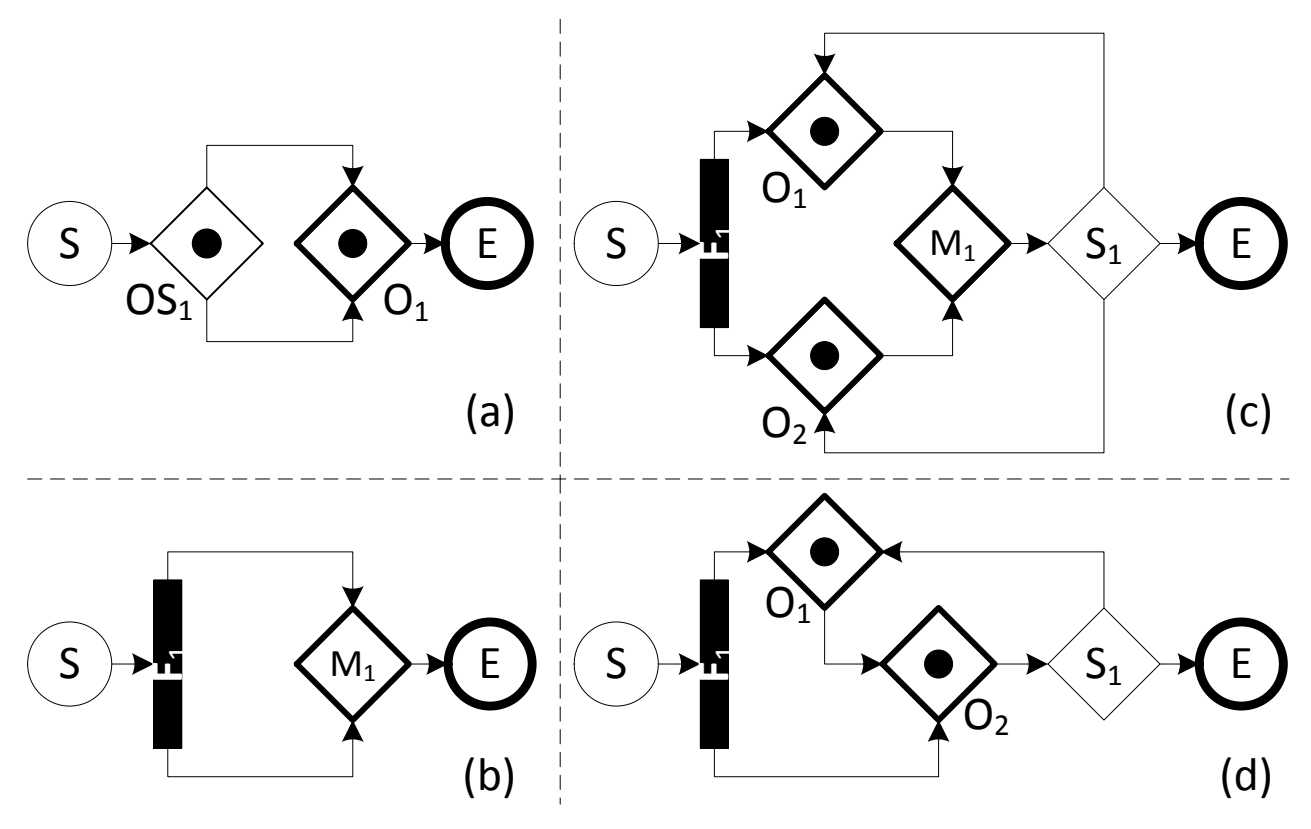

Figure 4. Sound and unsound workflow graphs

(1) No OR-join is enabled: $S$ would be a deadlock. \{

(2) OR-join $O_{1}$ is enabled and $O_{2}$ is not. As a consequence, $O_{1}$ fires and $O_{2}$ gets a token via its upper incoming edge $e_{4}$. Therefore, $\mathrm{O}_{2}$ synchronizes both tokens on its incoming edges to a single one on its outgoing edge. As a result, no deadlock and no state with a lack of synchronization is actually possible afterwards. $\checkmark$

(3) OR-join $O_{2}$ is enabled and $O_{1}$ is not. However, after $O_{2}$ has been fired, the split $S_{1}$ may decide to put a token on the incoming edge $e_{8}$ of the end node. If the other token eventually reaches $e_{8}$ (which is the case since the termination state of this workflow graph must have only one token on $e_{8}$ ), each termination state has a lack of synchronization. $\not$

(4) Both OR-joins are enabled and fire simultaneously. However, this concurrent execution may result in the same situation as in possibility (3). \{

In summary, only possibility (2) results in a sound behavior. There is a (yet undefined) release function for which our example is sound by Definition 1.

A possible release function $F_{\text {example }}$ holding for that example evaluates to enabled in a state $S$ for one of the both OR-joins $j$ iff

1. there is at least one incoming edge of $j$ which carries a token in $S$ and

2. a. if $j=O_{1}$ and none of the edges $e_{0}, e_{5}, e_{6}$, and $e_{8}$ carry a token in $S$

b. if $j=O_{2}$ and none of all edges except $e_{3}$ and $e_{4}$ carry a token in $S$.

We compare three popular OR-join release functions in the following. For this, we assume an OR-join $j$ and a state $S$ of an arbitrary workflow graph $W F G$ for the definitions of release functions.

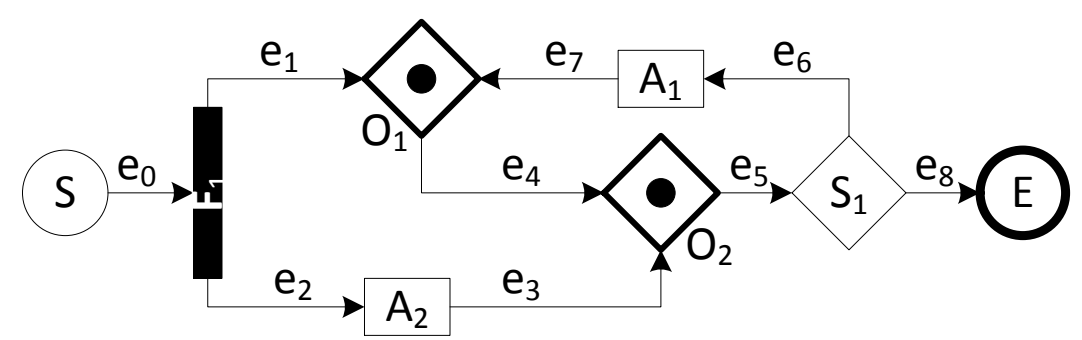

Figure 5. Workflow graph of the process in Figure $1(b)$ 
At first, we start with a state-space based approach of Kindler [3] that essentially considers all reachable states. As mentioned before, to consider all reachable states, we have to know the release function of OR-joins. However, to define this release function, we have to consider all reachable states. This is a cyclic dependency and it prevents a straight-forward formalization of the release function. Kindler solved this problem with the fix-point theory. At this point, we accept that Kindler has found a mathematical sound formalization. Then, the release function $F_{\text {Kindler }}$ evaluates to enabled iff

1. at least one incoming edge of $j$ carries a token in $S$ and

2. there is no token except the tokens on $j$ itself which can travel in a reachable state of $S$ to an incoming edge of $j$ that carries no token in $S$.

With this in mind, we see that our counter example leads to a deadlock since the token of $O_{1}$ may travel to $\mathrm{O}_{2}$ if $\mathrm{O}_{1}$ fires first or vice versa (cf. possibility (1)). Therefore, the semantics of Kindler cannot be complete.

Dumas et al. [6] follow a graph-based approach. They define their release function recursively. It evaluates to enabled for $j$ in $S$ in simple terms iff $j$ has an incoming edge which carries a token in $S$ and none of $j$ 's direct predecessor nodes, e.g., $p$, without a token on the edge $(p, j)$ has a (indirect) predecessor which is enabled. To avoid arbitrary recursion depths, each second enabledness evaluation of an indirect predecessor node $p^{\prime}$ of $p$ always evaluates to not enabled. Therefore, the release function $F_{\text {Dumas }}$ contains an additional set $V$ of visited nodes. $F_{\text {Dumas }}(j, S, V)$ evaluates to enabled with the function call $F_{\text {Dumas }}(j, S, \emptyset)$ iff

1. $j \notin V$ and there is an incoming edge $e$ of $j$ with $S(e) \geq 1$ and

2. for each edge $e^{\prime}=(n, j)$ with $S\left(e^{\prime}\right)=0$ exists no predecessor $p$ of $n$ for which $F_{\text {Dumas }}(p, S, V \cup\{j\})$ is enabled

Every time two OR-joins cannot be enabled until the other is not (cf. our counter example), both are treated as enabled. Thus, they are executed non-deterministically such that $O_{1}$ will be executed before $O_{2}$ or $O_{2}$ will be executed before $O_{1}$. That may result in a state with a lack of synchronization (cf. possibility (3) and (4)). For this reason, $F_{\text {Dumas }}$ is not complete.

Christiansen et al. [9] directly follow the BPMN 2.0 specification [1], i.e., they follow the approach of Völzer [2] as it is part of the current BPMN specification. The release function $F_{\text {Volzer }}$ evaluates to enabled for $j$ in state $S$ iff

1. there is an incoming edge $e$ of $j$ with $S(e) \geq 1$ and

2. each other edge $e^{\prime}$ of $W F G$ with $S\left(e^{\prime}\right) \geq 1$ having a path to an incoming edge of $j$ has also a path to $e$ without passing $j$

The argumentation of Völzer is that — in simple terms - if there is a possibility that a token reaches an incoming edge $e$ of $j$ which already carries a token, then $j$ should be fired first to avoid a lack of synchronization. Although this argumentation is comprehensible, our sound workflow graph in Figure 5 would lead to a deadlock since the token of $O_{1}$ can travel to the tokenless incoming edge $e_{4}$ of $\mathrm{O}_{2}$ and vice versa. Therefore, both OR-joins are not enabled. Völzer himself has already named that effect in his paper.

In summary, no considered OR-join release function is complete and therefore nobody can execute all sound workflow graphs successfully — there is the need for a most liberal release function. 


\section{A Complete OR-Join Semantics}

In the remainder of this section, we handle AND-joins as a special case of OR-joins for which tokens always arrive at all incoming edges. This is possible in sound workflow graphs as ANDjoins cause no deadlocks. For this, we refer to all OR- and AND-joins as OR-joins.

In the former sections, we have evaluated existing release functions. Since there exists no complete one, we want to derive our own approach for the first complete theory. Hence, we start at a common property of most release functions: An OR-join $j$ with a token on one of its incoming edges is not enabled since it has to "wait" for a further event - in general that no more tokens can ever arrive at an incoming edge of $j$. We formulate this "waiting" of an OR-join as follows:

Definition 3 (Waiting). Let $W F G=(N, E)$ be a sound workflow graph using a release function $F, j$ be an OR-join, and e be not an incoming edge of $j$.

$$
j \text { waits for e in state } S \text { iff }
$$

1. $j$ is active,

2. e carries a token, and

3. there is at least one path $P$ from e to an incoming edge of $j$, such that no node touched by $P$ prevents the token on efrom travelling via $P$ to $j$ (without firing $j$ ).

$j$ waits for node $n$ iff $e$ is an incoming edge of $n . j$ always waits for e iff in each state, where $j$ is active and e carries a token, $j$ waits for $e . j$ never waits for e iff in each such state $j$ does not wait for $e$.

Actually, this definition is very general: An OR-join $j$ will not be reached by a token on an edge $e$ in a state $S$ for two cases: Either (1) there is actually no path from $e$ to an incoming edge of $j$ or (2) on all these paths, the token would be prevented from traveling to $j$. It is simple to determine whether there is a path from $e$ to an incoming edge of $j$. The interesting nodes are those which prevent a token from travelling on a path from one edge to another edge. In the following, we introduce the basic property of nodes always preventing the traveling of tokens. As a consequence of this property, such nodes can only be OR-joins and they can only prevent the travel of tokens within cyclic workflow graphs. In such graphs, there are sometimes situations in which OR-joins seem to mutually wait for each other and therefore would cause a deadlock. With this in mind, we subsequently define an appropriate execution order for such situations deciding which OR-joins should wait and which OR-joins must not wait. As a result of these considerations, we derive our approach for a complete release function which we prove to be complete in conclusion.

\subsection{Basic Property}

As mentioned before, we introduce the basic property of a node $n$ always preventing the traveling of tokens from an edge $e$ to an incoming edge of an OR-join $j$. If such a node $n$ exists on all possible paths from $e$ to an incoming edge of $j, j$ does never have to wait for $e$. The following lemma describes that property which holds in this respect for sound workflow graphs.

Lemma 1. Let $W F G=(N, E)$ be a sound workflow graph, $j$ be an active OR-join in a state $S$, and e be an edge carrying a token in $S$.

$$
j \text { never waits for } e
$$

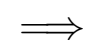

On all paths from e to an incoming edge of $j$ without passing $j$, an OR-join always waiting for $j$ can be activated 


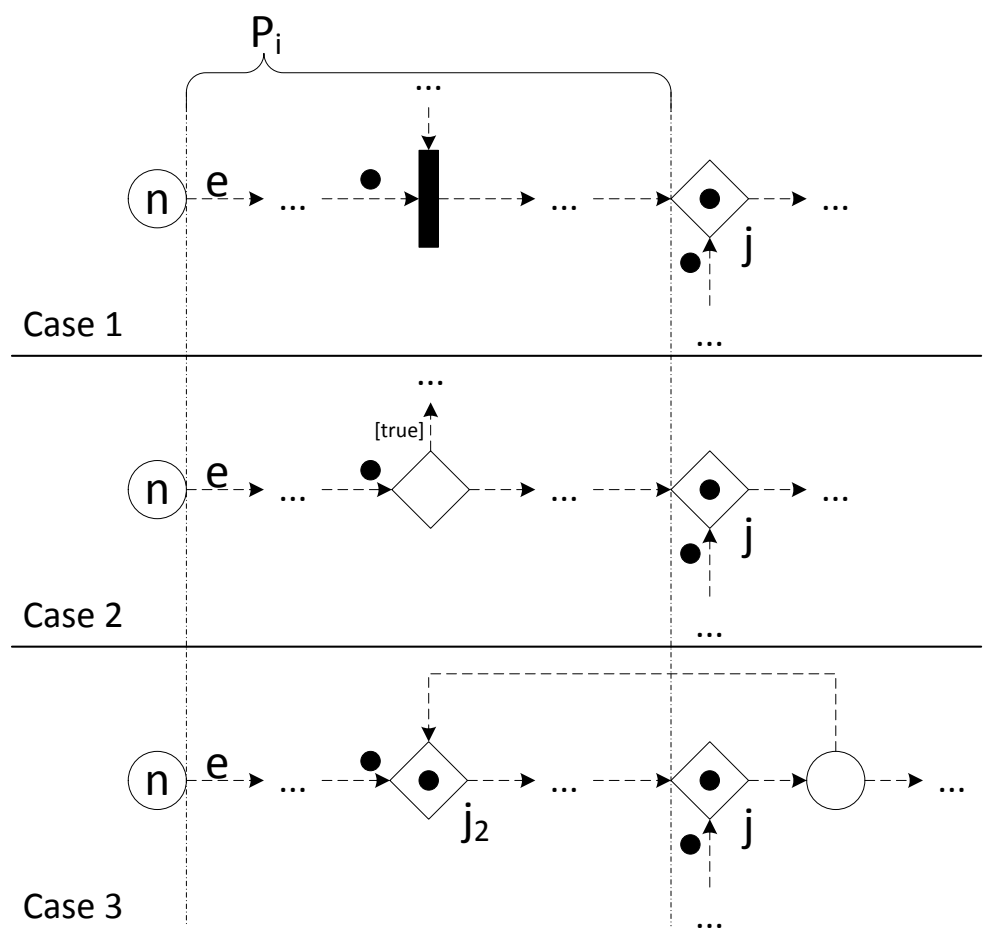

Figure 6. Cases how a token can never arrive at $j$

Proof (Lemma 1). There are two possibilities of $j$ never having to wait for $e$ :

1: There is not a path from $e$ to an incoming edge of $j$ without passing $j . \checkmark$

2: There are paths from $e$ to an incoming edge of $j$ without passing $j$.

Let $P_{i}$ be one of those paths $\stackrel{\text { Def. } 3}{\Longrightarrow}$ a token from $e$ can never arrive at $j$ via $P_{i}$ in a reachable state since otherwise $j$ would wait for $e \Rightarrow$ the token either (1) is blocked on $P_{i}$, (2) always leaves $P_{i}$, or (3) activates a node on $P_{i}$ which always waits for $j$ (cf. Figure 6).

Case 1: The token is blocked on $P_{i} \Rightarrow$ a deadlock must have occurred. \{

Case 2: The token always leaves $P_{i} \Rightarrow$ there is a split or an OR-split on $P_{i}$ which always decides that the token does not travel on $P_{i}$. Since splits and OR-splits decide nondeterministically, this case is impossible. \{

Case 3: The token activates a node on $P_{i}$ which always waits for $j$. This node must be an ORjoin $j_{2}$ since it must be able to wait. Since there would be a deadlock if $j$ also waits for $j_{2}, j$ never has to wait for $j_{2} \cdot \checkmark$

In the next lemma, we show that the inversion holds too.

Lemma 2. Let $W F G=(N, E)$ be a sound workflow graph, $j$ be an active OR-join in a state $S$, and $e$ be an edge carrying a token in $S$.

On all paths from e to an incoming edge of $j$ without passing $j$, an OR-join always waiting for $j$ can be activated

$$
\text { j never waits for } e
$$


Proof (Lemma 2). Constructive proof.

Case 1: There is no a path from $e$ to an incoming edge of $j$ without passing $j \stackrel{\text { Def. } 3}{\Longrightarrow} j$ never waits for $e \checkmark$

Case 2: There are paths from $e$ to an incoming edge of $j$ without passing $j$ (see the figure on the right of this text) $\stackrel{\text { assumption }}{\Longrightarrow}$ if the token on $e$ travelled a path $P_{i}$ to $j$, it would have activated an OR-join $w_{i}$ which would always wait for $j$ $\stackrel{\text { deadlock-free }}{\Longrightarrow} j$ never waits for $w_{i}$

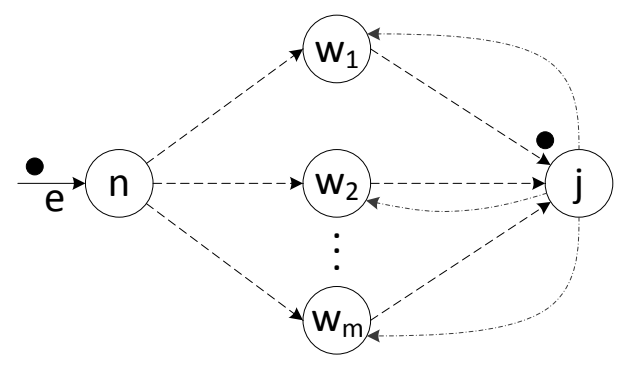

$\Longrightarrow j$ can always be executed before $w_{i}$

$\Longrightarrow j$ never waits for $e \checkmark$

Then, the following theorem formulates our basic property:

Theorem 1. Let $W F G=(N, E)$ be a sound workflow graph, $j$ be an active OR-join in a state $S$, and $e$ be an edge carrying a token in $S$.

$j$ never waits for $e$

\author{
On all paths from e to an incoming edge of $j$ without passing $j$, \\ an OR-join always waiting for $j$ can be activated
}

Proof (Theorem 1). Follows directly from Lemma 1 and 2.

\title{
5.2 Determination of an Appropriate Execution Order
}

In the case of acyclic sound workflow graphs, the definition of a release function is simply a result of the previous basic property: An OR-join $j$ only waits in state $S$ for all those edges carrying a token and having a path to an incoming edge of $j$. Otherwise, there must be a cycle.

In contrast, in the cyclic case, two OR-joins may mutually wait for each other and therefore may cause a deadlock: A vicious circle [3]. In order to avoid these circles, the definition of a release function has to describe in principle the appropriate execution order deciding which ORjoin in such circles has to wait and which OR-join must not wait. A concept of compiler theory helps to find an important property in which an OR-join always waits for a token on an edge the post-dominance relation.

Definition 4 (Post-Dominance). Let $W F G=(N, E)$ be a workflow graph with its end node end.

A node $n$ post-dominates node $m$ if every path from $m$ to end contains $n$, written $n$ pdom $m$. $n$ properly post-dominates $m$ if $n$ pdom $m$ and $n \neq m$. $n$ directly post-dominates $m$ if $n$ properly post-dominates $m$ and any other node o that properly post-dominates $m$ also properly postdominates $n$, i.e., the direct post-dominance relation is transitive.

A post-dominator tree of $W F G$ is a directed graph pdom $(W F G)=\left(N, E^{\prime}\right)$ with $E^{\prime}=\{(n, m): n$ directly post-dominates $m$ in $W F G\}$.

Figure 7 shows the sample workflow graph $(a)$ of Figure 5 and its post-dominator tree $(b)$.

In sound workflow graphs, the post-dominance relation guarantees that a post-dominator $j$ of an active node $n$ will be executed in each case. Hence, such a token on an incoming edge of $n$ travels eventually on a path to the incoming edge of the end node (and so via $j$ ) to be consumed in the termination state. The next theorem uses this fact and describes an important property in which an OR-join always waits for nodes which it post-dominates. 

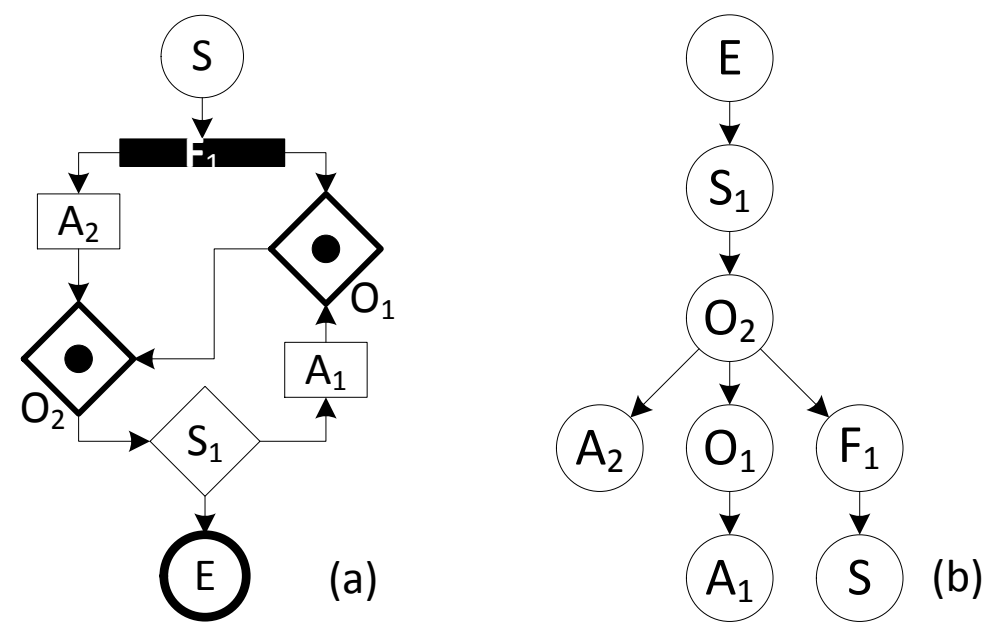

Figure 7. The workflow graph $(a)$ of Figure 5 and its post-dominator tree $(b)$

Theorem 2. Let $W F G=(N, E)$ be a sound workflow graph, $j$ be an active OR-join in a state $S$, and $n$ another active node in $S$.

$$
\begin{gathered}
j \text { lies on all paths from } n \text { to the end node }(j \text { pdom } n) \\
\Longrightarrow \\
j \text { always waits for } n
\end{gathered}
$$

Proof (Theorem 2). Let $p \operatorname{dom}(W F G)$ be the post-dominator tree of $W F G$. It defines a partial order caused by the post-dominance relation.

We use this order to assign numbers to each OR-join (cf. the figure on the right of this text): Each OR-join $j$ is given the number of OR-joins lying on the path from $j$ to the root node, e.g., an OR-join labelled $d$ has $d$ predecessors being OR-joins in $\operatorname{pdom}(W F G)$. We write $j_{d}$ for an OR-join $j$ with $d$ predecessors being OR-joins in $p d o m(W F G)$. We can reformulate the hypothesis of this lemma as follows if $j_{d}$ is an active OR-join in state $S$ and $n$ is another active node in $S$ :

$$
j_{d} \text { pdom } n \quad \Longrightarrow \quad j_{d} \text { always waits for } n
$$

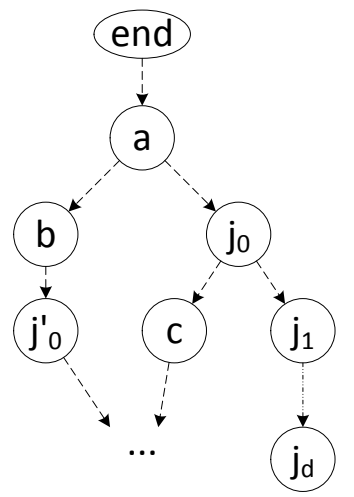

This proposition will be proven by mathematical induction and by contradiction. Therefore, we start with $d=0$.

Assumption: $j_{0}$ does not have to wait for $n$ in a state $S$ $\Rightarrow j_{0}$ can be executed independently from $n$ in a state $S_{1}$, $S \rightarrow^{*} S_{1}$.

Assume $j_{0}$ is executed before $n$ in $S_{1}$

$\Rightarrow$ the end node can be activated in a subsequent state $S_{2}$, $S_{1} \rightarrow{ }^{*} S_{2}$, since there is a path from $j_{0}$ to end without another

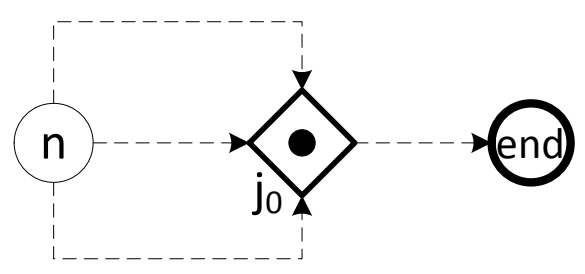
OR-join

$\Rightarrow e n d$ and $n$ are active in $S_{2}$

$\Rightarrow S_{2}$ has a lack of synchronization when the token from the outgoing edge of $n$ eventually arrives at the incoming edge of end. \{ 
The induction step is based on the hypothesis being valid for $j_{d}$. So we have to show that it holds for $j_{d+1}$. The figure on the righthand side reflects the overall situation.

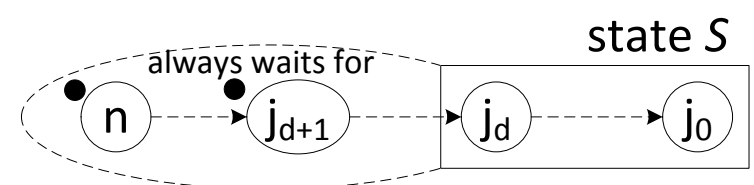

Assumption: $j_{d+1}$ does not have to wait for $n$ in a state $S$

$\Rightarrow j_{d+1}$ can be executed independently from $n$ in a state $S_{1}, S \rightarrow^{*} S_{1}$.

Assume $j_{d+1}$ is executed before $n$ in $S_{1}$

$\Rightarrow j_{d}$ can be activated in a following state $S_{2}, S_{1} \rightarrow^{*} S_{2}$ (since there is a path $P$ from $j_{d+1}$ to $j_{d}$ without another OR-join).

Assume $j_{d}$ is activated in $S_{2}$ and $n$ has not fired yet

$\stackrel{\text { hypothesis }}{\Rightarrow} j_{d}$ always waits for $n$

$\stackrel{\text { deadlock-free }}{\Rightarrow} n$ never waits for $j_{d}$

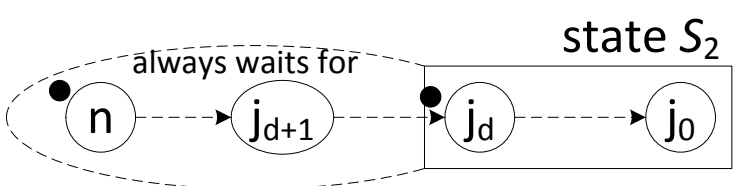

$\Rightarrow$ the token on the outgoing edge of $n$ can arrive at $j_{d+1}$ in a state $S_{3}, S_{2} \rightarrow^{*} S_{3}$, after $n$ has been fired before $j_{d}$.

Assume $j_{d+1}$ is again activated and it is active at the same time as $j_{d}$ in $S_{3}$

$\stackrel{\text { hypothesis }}{\Rightarrow} j_{d}$ always waits for $j_{d+1}$ since $j_{d}$ postdominates $j_{d+1}$

$\stackrel{\text { deadlock-free }}{\Rightarrow}$ the same path $P$ from $j_{d+1}$ to $j_{d}$ can be traveled once more by the token of the outgoing edge of $j_{d+1}$ after firing $j_{d+1}$

$\Rightarrow$ there is a state $S_{4}, S_{3} \rightarrow^{*} S_{4}$, with a lack of synchronization at an incoming edge of $j_{d} \cdot\{$

As a result, the following corollary is directly derived from the previous theorem since two OR-joins mutually waiting for each other would cause a deadlock.

Corollary 1. Let $W F G=(N, E)$ be a sound workflow graph, $j$ be an active OR-join in a state $S$, and $n$ another active node in $S$.

$$
\begin{gathered}
n \text { is post-dominated by } j \\
\Longrightarrow \\
n \text { never waits for } j
\end{gathered}
$$

In principle, Corollary 1 shows that in vicious circles, an OR-join does not have to wait for its post-dominator OR-joins. For now, we assume that vicious circles have only an appropriate execution order if one OR-join post-dominates the other. The correctness of this assumption, i.e., the completeness of our release function, will be proven afterwards in Theorem 3. Under these terms, the following definition that results, in particular, from the previous corollary describes our approach of a release function of an OR-join.

Definition 5 (Release Function). Let $W F G=(N, E)$ be a sound workflow graph and $j$ be an OR-join.

The release function $F_{\text {compl }}$ evaluates to enabled for $j$ in a state $S$ iff

1. there is an incoming edge e of $j$ with $S(e) \geq 1$ and

2. on each path $P$ without passing $j$ from an active node $n$ in $S$ to $j$ lies an OR-join which post-dominates $j$ 
As a result of our property (Theorem 1), this definition implicitly makes use of the fact that an active OR-join $j$ in a state $S$ never waits for an edge $e$ carrying a token if there is an OR-join post-dominating $j$ on all paths from $e$ to that $j$.

Since we now have defined our approach of a release function, we can apply it to our counter example (cf. Figure 5) of Section 4. The situation is the same: Both OR-joins $O_{1}$ and $O_{2}$ are active in a reachable state $S$ from the initial state. If we apply our semantics, OR-join $O_{2}$ waits for $O_{1}$ and $O_{1}$ is enabled to fire since $O_{2}$ post-dominates $O_{1}$. It results in the desired behavior of possibility (2) (cf. Section 4) describing that $O_{2}$ will be executed before $O_{1}$ if both are active in the same state. In this case and as mentioned before in Section 4, this execution order leads to a sound behavior.

\subsection{Completeness of our Approach}

Our assumption was that vicious circles only can be resolved if one of the OR-joins post-dominates the other. If that holds, our release function would be complete. In the following theorem, it will be proven that this assumption is correct, i.e., there is no appropriate execution order otherwise.

Theorem 3 (Completeness). Let $W F G=(N, E)$ be a workflow graph by using an arbitrary release function $F$ and $j_{1}, j_{2}$ be two different active OR-joins in a state $S$ which have at least one path to one another.

\section{$j_{1}$ does not post-dominate $j_{2}$ and $j_{2}$ does not post-dominate $j_{1}$

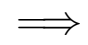 \\ WFG is unsound}

Proof (Theorem 3). Constructive proof.

Case a: $j_{1}$ always waits for $j_{2}$ and $j_{2}$ always waits for $j_{1}-$ a deadlock. $\checkmark$

Case b: $j_{1}$ does not wait for $j_{2}$ or $j_{2}$ does not wait for $j_{1}$ in state $S$. Without loss of generality, we assume that $j_{2}$ does not wait for $j_{1} \Rightarrow j_{2}$ is executed before or at the same time as $j_{1}$. Furthermore, there is at least one path $P^{\prime}$ from the incoming edge of $j_{2}$ carrying a token in $S$ to the incoming edge of the end node without an incoming edge of $j_{1}$ (since $j_{1}$ does not post-dominate $j_{2}$ ). There is

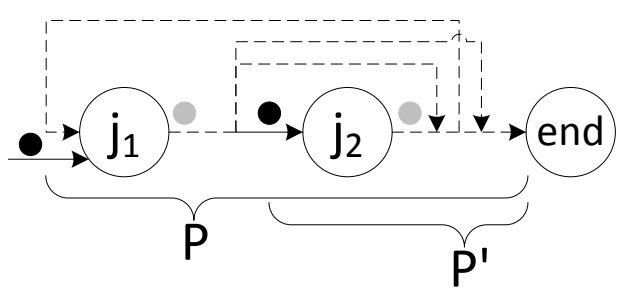
also one path $P$ from the incoming edge of $j_{1}$ carrying a token in $S$ to the incoming edge of the end node where $P$ contains $P^{\prime}$ as a subpath since there is a path from $j_{1}$ to $j_{2}$ (cf. conditions of this Theorem 3). The figure above illustrates the situation conceptually.

Since $j_{2}$ does not wait for $j_{1}$ in state $S$ as mentioned before, let $j_{2}$ be executed before or at the same time as $j_{1}$ in a reachable state $S_{1}$. As a consequence, in both cases, $j_{2}$ is executed and its outgoing edge carries a token in $S_{1}$. A possible state $S_{1}$ of an execution, which, e.g., arose from a concurrent execution of $j_{1}$ and $j_{2}$, is illustrated in the figure above with grey dots.

Now, we assume a variable $S_{c}$ for the current state (initially $S_{c}$ is $S_{1}$ ) and a variable $x$ which initially represents the incoming or outgoing edge of $j_{1}$ (depending on whether $j_{1}$ was executed before state $S_{1}$ or not) and which describes the position on path $P$ of the "token of

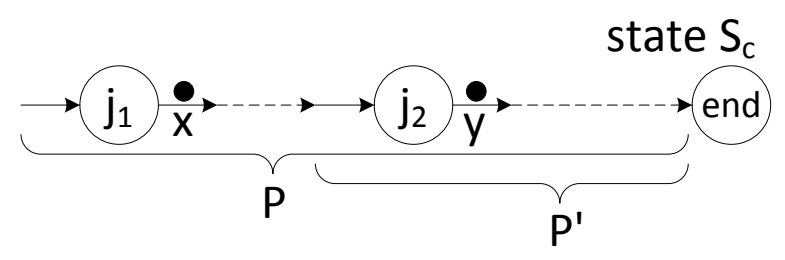
$j_{1}$ " in the current state $S_{c}$. Furthermore, there is a variable $y$ which initially is the outgoing edge of $j_{2}$ and which represents the position on the same path $P$ of the "token of $j_{2}$ " in $S_{c}$.

In $S_{c}$, there always exists two possibilities for the nodes $n_{x}$ and $n_{y}$ being currently activated by the edges $x=\left(n^{\prime}, n_{x}\right)$ and $y=\left(n^{\prime \prime}, n_{y}\right)$ :

Case 1: $n_{x}$ cannot be executed and $n_{y}$ cannot be executed since $n_{x}$ always waits for $n_{y}$ and $n_{y}$ always waits for $n_{x} \Rightarrow$ deadlock 


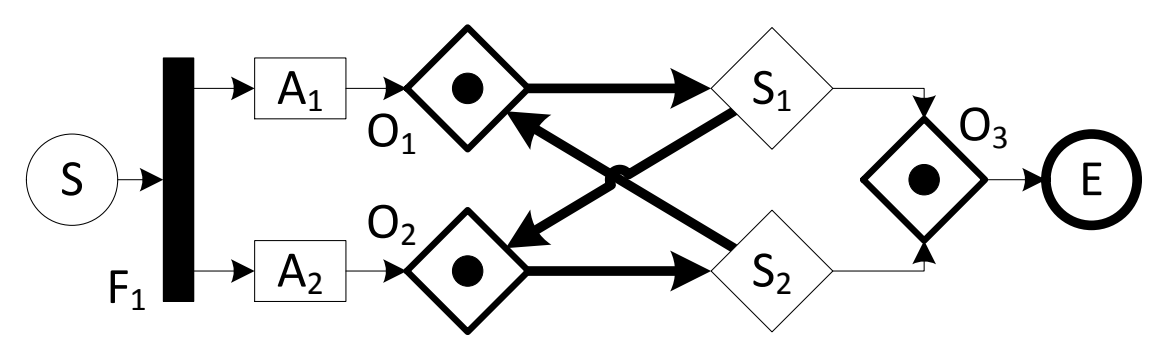

Figure 8. A workflow graph with two OR-joins mutually waiting for each other

Case 2: $n_{x}$ can be executed or $n_{y}$ can be executed in $S_{c}$ (possibly at the same time) $\Rightarrow$ $n_{x}$ or $n_{y}$ will be executed, tokens could be put on that outgoing edges of $n_{x}$ (if $n_{x}$ was executed) or $n_{y}$ (if $n_{y}$ was executed) which lie on

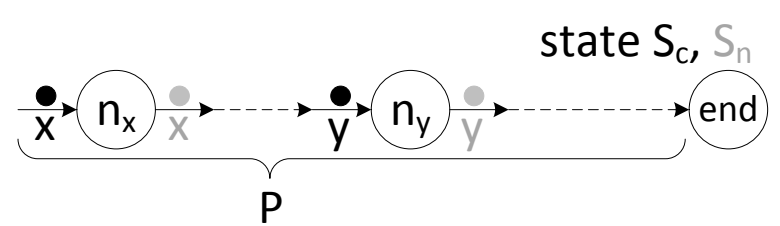
path $P$, the followed state is $S_{n} \Rightarrow$ afterwards, let $S_{c}$ be $S_{n}$ and $x$ and $y$ those edges on $P$ now carrying a token, i.e., the mentioned outgoing edges of $n_{x}$ and $n_{y}$.

Both cases show that there is either a deadlock or both tokens can exclusively travel on possibly different edges $x$ and $y$ of path $P$. Since both tokens on $x$ and $y$ must eventually arrive at the same edge of path $P^{\prime}$ in future $(x=y)$, this would lead to a state with a lack of synchronization. All cases results in an unsound behavior. $\checkmark$

In all cases, an execution could result in a deadlock or in a state with a lack of synchronization. Hence, the workflow graph must be unsound! $\checkmark$

In summary, our proposed release function fits in acyclic cases since active OR-joins always have to wait for tokens on edges which have paths to them (Theorem 1). In the cyclic case, only vicious circles prevent a simple definition of a release function as deadlocks occur (caused by mutually waiting OR-joins). Such situations can only be resolved if one OR-join post-dominates the other as mentioned in Theorem 3 - otherwise, a deadlock or a state with a lack of synchronization is possible. In short, our approach must be complete as all cases are handled.

The following example shows a workflow graph which has a deadlock caused by two mutually waiting OR-joins - also by using our complete release function. Figure 8 shows this workflow graph with a vicious circle $O_{1} \rightarrow S_{1} \rightarrow O_{2} \rightarrow S_{2} \rightarrow O_{1}$ and demonstrates that deadlocks actually are not exclusive phenomenons of AND-joins: After the execution of $F_{1}$, both OR-joins $O_{1}$ and $\mathrm{O}_{2}$ are activated and therefore, $\mathrm{O}_{1}$ waits for $\mathrm{O}_{2}$ and vice versa since neither $O_{1}$ post-dominates $O_{2}$ nor $O_{2}$ post-dominates $O_{1}$ and there are paths to each other. This state is a deadlock. As stated in Theorem 3, the workflow graph is unsound. For example, if we allow one or both of the OR-joins to be executed, the two tokens may arrive at the same incoming edge of the OR-join $\mathrm{O}_{3}$.

\section{Semantics in Practice}

After introducing and proving our complete release function - and therefore the most liberal semantics of OR-joins - , we show how that semantics can be applied in practice. For this purpose, we use the realistic sample process of Figure 2 as shown once again with labels in Figure 9.

We imagine a reachable state $S$ in which the edges $e_{4}, e_{15}$, and $e_{16}$ carry a token as illustrated in the figure with black dots. One token (on $e_{4}$ ) activates the OR-join $O J_{2}$, another token on $e_{15}$ activates the OR-join $O J_{1}$. Existing OR-join semantics now may lead to a deadlock or to a state with a lack of synchronization as mentioned in Section 4. Applying our semantics, however, makes the process in each case executable in failure-free way. Therefore, at first, we determine the postdominator tree of our sample process (cf. Figure 10). 


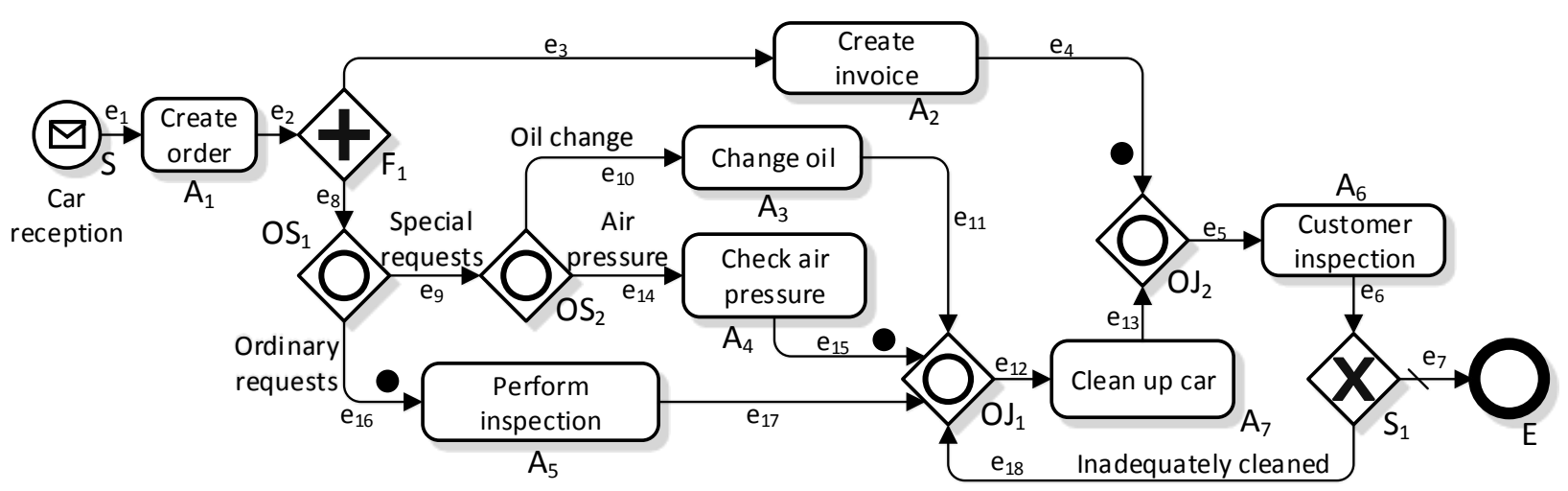

Figure 9. Car service process with labels

The tree shows that OR-join $O J_{2}$ post-dominates $O J_{1}$. Therefore, on each path from the edge $e_{4}$ to an incoming edge of the OR-join $O J_{1}$, OR-join $O J_{2}$ is passed, i.e., $O J_{1}$ does not wait for the token of the edge $e_{4}$ (cf. Def. 5). As a result, $O J_{1}$ would wait for possible tokens on the edges $e_{1}$, $e_{2}, e_{5}, e_{6}, e_{8}, e_{9}, e_{10}, e_{14}, e_{16}$, and $e_{18}$; and therefore waits in the current state $S$ for the token of $e_{16}$. On the other hand, $O J_{2}$ waits for all edges currently carrying a token as there is no OR-join that directly post-dominates $O J_{2}$, i.e., $O J_{2}$ would wait for possible tokens on all edges except $e_{4}$, $e_{7}$, and $e_{13}$. As we have proven, the process would never produce a failure situation and leads to a sound behavior.

The explanation of the previous example shows furthermore that the set of edges $\omega(j)$ an active OR-join $j$ would wait for when one of those edges carries a token can be precomputed before an execution of a process. If we assume that we use a state representation which is a subset of the set of edges carrying a token, then the evaluation whether an active OR-join $j$ can be executed or not in a state $S$ is possible in constant time at runtime $(S \cap \omega(j)=\emptyset)$.

Another example is illustrated in Figure 11 which shows a structured process with two parallel OR-split and OR-joins within a loop. We use that example to show that our OR-join semantics also works with AND-joins since we handle them like OR-joins as mentioned at the beginning of Section 5. For this reason, the AND-join $J$ post-dominates both OR-joins $O J_{1}$ and $O J_{2}$, i.e., both do not wait for one another with regard to our release function. Therefore, the process will be executed as intended.

\section{Conclusion}

In this paper, we have presented a new definition of soundness for workflow graphs with arbitrary OR-join semantics. Based on this definition, we introduced a quality criterion - completeness — to evaluate semantics of research, especially the semantics of Völzer [2]. Afterwards, a new approach was determined which was proven to be complete. We have shown, that it is (to the best

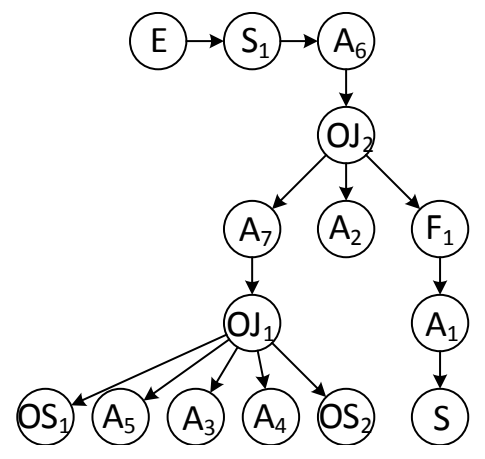

Figure 10. The post-dominator tree of the process of Figure 9 


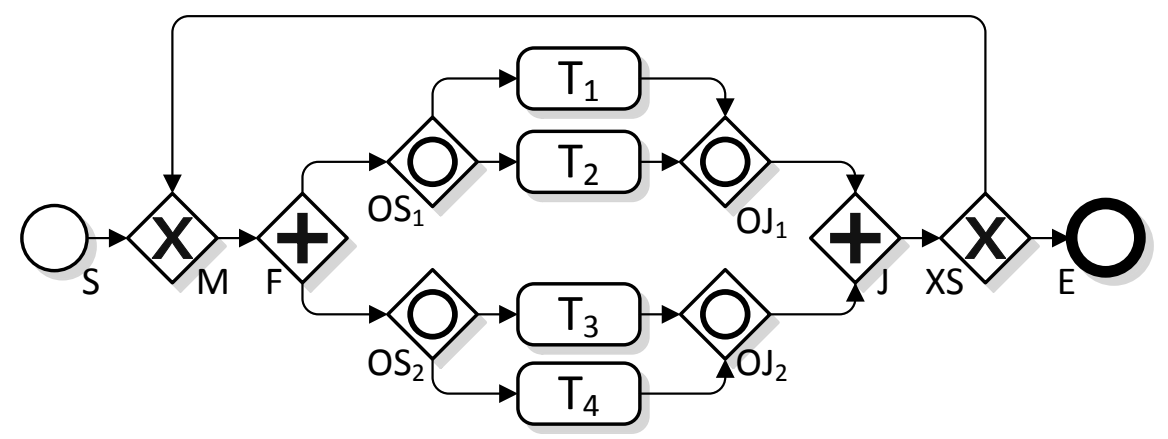

Figure 11. A structured process with an AND-join

author's knowledge) the first, most liberal OR-join semantics which holds for all sound workflow graphs. At the end, it could be shown that two or more OR-joins can actually cause a deadlock in unsound workflow graphs when no correct execution order exists. Our proposed semantics helps to detect such failure situations by static analysis.

Our next step in future work will be to develop a first approach for detecting all deadlocks and states with lack of synchronization within processes containing OR-joins. Hence, we have to extend previous work, e.g., Vanhatalo et al. [14], [16], Fahland et al. [17], and our previous work for process analyses and execution [13], [18], [19], [20].

\section{References}

[1] OMG, "Business Process Model and Notation 2.0," formal/2011-01-03, 2011. [Online]. Available: http://www.omg.org/spec/BPMN/2.0

[2] H. Völzer, "A new semantics for the inclusive converging gateway in safe processes," in Business Process Management - 8th International Conference, BPM 2010, Hoboken, NJ, USA, September 13-16, 2010. Proceedings, ser. Lecture Notes in Computer Science, R. Hull, J. Mendling, and S. Tai, Eds., vol. 6336. Springer, 2010, pp. 294-309. [Online]. Available: http://dx.doi.org/10.1007/978-3-642-15618-2_21

[3] E. Kindler, "On the semantics of EPCs: A framework for resolving the vicious circle," in Business Process Management: Second International Conference, BPM 2004, Potsdam, Germany, June 17-18, 2004. Proceedings, ser. Lecture Notes in Computer Science, J. Desel, B. Pernici, and M. Weske, Eds., vol. 3080. Springer, 2004, pp. 82-97. [Online]. Available: http://dx.doi.org/10.1007/978-3-540-25970-1_6

[4] E. Kindler, "On the semantics of EPCs: Resolving the vicious circle," Data Knowl. Eng., vol. 56, no. 1, pp. 23-40, 2006. [Online]. Available: http://dx.doi.org/10.1016/j.datak.2005.02.005

[5] J. Mendling and W. M. P. van der Aalst, "Formalization and verification of EPCs with OR-joins based on state and context," in Advanced Information Systems Engineering, 19th International Conference, CAiSE 2007, Trondheim, Norway, June 11-15, 2007, Proceedings, ser. Lecture Notes in Computer Science, J. Krogstie, A. L. Opdahl, and G. Sindre, Eds., vol. 4495. Springer, 2007, pp. 439-453. [Online]. Available: http://dx.doi.org/10.1007/978-3-540-72988-4_31

[6] M. Dumas, A. Großkopf, T. Hettel, and M. T. Wynn, "Semantics of standard process models with OR-joins," in On the Move to Meaningful Internet Systems 2007: CoopIS, DOA, ODBASE, GADA, and IS, OTM Confederated International Conferences CoopIS, DOA, ODBASE, GADA, and IS 2007, Vilamoura, Portugal, November 25-30, 2007, Proceedings, Part I, ser. Lecture Notes in Computer Science, R. Meersman and Z. Tari, Eds., vol. 4803. Springer, 2007, pp. 41-58. [Online]. Available: http://dx.doi.org/10.1007/978-3-540-76848-7_5 
[7] N. Cuntz and E. Kindler, "On the semantics of epcs: Efficient calculation and simulation," in Business Process Management, 3rd International Conference, BPM 2005, Nancy, France, September 5-8, 2005, Proceedings, W. M. P. van der Aalst, B. Benatallah, F. Casati, and F. Curbera, Eds., vol. 3649, 2005, pp. 398-403. [Online]. Available: http://dx.doi.org/10.1007/11538394_30

[8] M. T. Wynn, D. Edmond, W. M. P. van der Aalst, and A. H. M. ter Hofstede, "Achieving a general, formal and decidable approach to the OR-join in workflow using reset nets," in ICATPN, ser. Lecture Notes in Computer Science, G. Ciardo and P. Darondeau, Eds., vol. 3536. Springer, 2005, pp. 423443.

[9] D. R. Christiansen, M. Carbone, and T. T. Hildebrandt, "Formal semantics and implementation of BPMN 2.0 inclusive gateways," in WS-FM, ser. Lecture Notes in Computer Science, M. Bravetti and T. Bultan, Eds., vol. 6551. Springer, 2010, pp. 146-160. [Online]. Available: http://dx.doi.org/10.1007/978-3-642-19589-1

[10] W. M. P. van der Aalst and A. H. M. ter Hofstede, "Yawl: yet another workflow language," Inf. Syst., vol. 30, no. 4, pp. 245-275, 2005.

[11] W. Sadiq and M. E. Orlowska, "Analyzing process models using graph reduction techniques," Inf. Syst., vol. 25, no. 2, pp. 117-134, 2000. [Online]. Available: http://dx.doi.org/10.1016/ S0306-4379(00)00012-0

[12] W. M. P. van der Aalst, A. Hirnschall, and H. M. W. E. Verbeek, "An alternative way to analyze workflow graphs," in Advanced Information Systems Engineering, 14th International Conference, CAiSE 2002, Toronto, Canada, May 27-31, 2002, Proceedings, ser. Lecture Notes in Computer Science, A. B. Pidduck, J. Mylopoulos, C. C. Woo, and M. T. Özsu, Eds., vol. 2348. Springer, 2002, pp. 535-552. [Online]. Available: http://link.springer.de/link/service/series/0558/bibs/2348/23480535.htm

[13] T. M. Prinz and W. Amme, "Practical compiler-based user support during the development of business processes," in Service-Oriented Computing - ICSOC 2013 Workshops - CCSA, CSB, PASCEB, SWESE, WESOA, and PhD Symposium, Berlin, Germany, December 2-5, 2013. Revised Selected Papers, ser. Lecture Notes in Computer Science, A. Lomuscio, S. Nepal, F. Patrizi, B. Benatallah, and I. Brandic, Eds., vol. 8377. Springer, 2013, pp. 40-53. [Online]. Available: http://dx.doi.org/10.1007/978-3-319-06859-6_5

[14] J. Vanhatalo, H. Völzer, and F. Leymann, "Faster and more focused control-flow analysis for business process models through SESE decomposition," in Service-Oriented Computing - ICSOC 2007, Fifth International Conference, Vienna, Austria, September 17-20, 2007, Proceedings, ser. Lecture Notes in Computer Science, B. J. Krämer, K. Lin, and P. Narasimhan, Eds., vol. 4749. Springer, 2007, pp. 43-55. [Online]. Available: http://dx.doi.org/10.1007/978-3-540-74974-5_4

[15] W. M. P. van der Aalst, "Verification of workflow nets," in Application and Theory of Petri Nets 1997, 18th International Conference, ICATPN '97, Toulouse, France, June 23-27, 1997, Proceedings, ser. Lecture Notes in Computer Science, P. Azéma and G. Balbo, Eds., vol. 1248. Springer, 1997, pp. 407-426. [Online]. Available: http://dx.doi.org/10.1007/3-540-63139-9_48

[16] J. Vanhatalo, H. Völzer, and J. Koehler, "The refined process structure tree," in Business Process Management, 6th International Conference, BPM 2008, Milan, Italy, September 24, 2008. Proceedings, ser. Lecture Notes in Computer Science, M. Dumas, M. Reichert, and M. Shan, Eds., vol. 5240. Springer, 2008, pp. 100-115. [Online]. Available: http: //dx.doi.org/10.1007/978-3-540-85758-7_10

[17] D. Fahland, C. Favre, J. Koehler, N. Lohmann, H. Völzer, and K. Wolf, "Analysis on demand: Instantaneous soundness checking of industrial business process models," Data Knowl. Eng., vol. 70, no. 5, pp. 448-466, May 2011. [Online]. Available: http://dx.doi.org/10.1016/j.datak.2011.01.004 
[18] T. M. Prinz, N. Spieß, and W. Amme, "A first step towards a compiler for business processes," in Compiler Construction - 23rd International Conference, CC 2014, ETAPS 2014, Grenoble, France, April 5-13, 2014. Proceedings, ser. Lecture Notes in Computer Science, A. Cohen, Ed., vol. 8409. Springer, 2014, pp. 238-243. [Online]. Available: http://dx.doi.org/10.1007/978-3-642-54807-9_14

[19] T. M. Prinz, "Proposals for a virtual machine for business processes," in Proceedings of the 7th Central European Workshop on Services and their Composition, ZEUS 2015, Jena, Germany, February 19-20, 2015., ser. CEUR Workshop Proceedings, T. S. Heinze and T. M. Prinz, Eds., vol. 1360. CEUR-WS.org, 2015, pp. 10-17. [Online]. Available: http://ceur-ws.org/Vol-1360/paper2.pdf

[20] T. M. Prinz, T. S. Heinze, W. Amme, J. Kretzschmar, and C. Beckstein, "Towards a compiler for business processes - a research agenda," in SERVICE COMPUTATION 2015: The Seventh International Conferences on Advanced Service Computing, M. de Barros and C.-P. Rückemann, Eds., vol. 6, IARIA Conference. Nice, France: ThinkMind Digital Library, March 22 2015, pp. 49-54. 